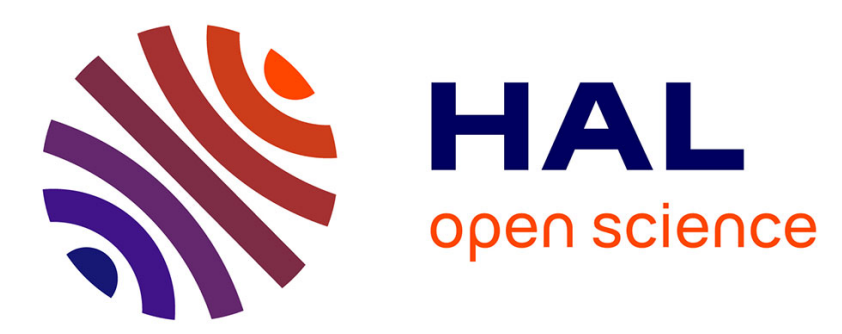

\title{
Estimates of Lithospheric Failure Cycle Parameters from Regional Earthquake Catalogues
}

\author{
V. Smirnov, S. Ommi, M. Potanina, V. Mikhailov, A. Petrov, N. M. Shapiro,
} A. Ponomarev

\section{- To cite this version:}

V. Smirnov, S. Ommi, M. Potanina, V. Mikhailov, A. Petrov, et al.. Estimates of Lithospheric Failure Cycle Parameters from Regional Earthquake Catalogues. Fizika zemli / Izvestiya Physics of the Solid Earth, 2019, 55 (5), pp.701-718. 10.1134/S1069351319050124 . hal-03088554

\section{HAL Id: hal-03088554 https://hal.science/hal-03088554}

Submitted on 15 Jan 2021

HAL is a multi-disciplinary open access archive for the deposit and dissemination of scientific research documents, whether they are published or not. The documents may come from teaching and research institutions in France or abroad, or from public or private research centers.
L'archive ouverte pluridisciplinaire HAL, est destinée au dépôt et à la diffusion de documents scientifiques de niveau recherche, publiés ou non, émanant des établissements d'enseignement et de recherche français ou étrangers, des laboratoires publics ou privés. 


\title{
Estimates of Lithospheric Failure Cycle Parameters from Regional Earthquake Catalogues
}

\author{
V. B. Smirnov ${ }^{a, b, c, *}$, S. Ommi ${ }^{d}$, M. G. Potanina ${ }^{a, b}$, V. O. Mikhailov ${ }^{a, b, c}$, \\ A. G. Petrov ${ }^{a, b}$, N. M. Shapiro ${ }^{b, c}$, and A. V. Ponomarev ${ }^{b}$ \\ ${ }^{a}$ Faculty of Physics, Moscow State University, Moscow, 119991 Russia \\ ${ }^{b}$ Schmidt Institute of Physics of the Earth, Russian Academy of Sciences, Moscow, 123242 Russia \\ ${ }^{c}$ Institut de Physique du Globe de Paris, Paris, 75005 France \\ ${ }^{d}$ International Institute of Earthquake Engineering and Seismology (IIEES), Tehran, 19537-14453 Iran \\ *e-mail:vs60@mail.ru
}

Received May 28, 2018; revised November 30, 2018; accepted December 18, 2018

\begin{abstract}
Based on the generalized frequency-magnitude relation for earthquakes, the failure cycle parameters are estimated from regional seismic catalogues for zones with different typical tectonic regimes: subduction zones and shear fault zones. The Kamchatka and Japan catalogues are used for the subduction zones. The estimates for the shear fault zones are based on the data for the San-Andreas Fault presented in the Northern California Earthquake Catalog, the data for the North Anatolian fault presented in the earthquake catalogue for Greece, and the data for the Zagros shear zone presented in the catalogue for Iran. Distribution maps are constructed for the failure cycle duration and for parameter $q$ characterizing the dependence of the duration of the failure cycle on the size of the fractured area. It is shown that on average for each region, the $q$ values are close to zero, indicating that the probability of failure for structural elements of the medium does not depend on their size and that the strength and stress distributions by size of inhomogeneities are, on average, consistent with each other. The intraregional spatial variations of parameter $q$ exceed the statistical error of the estimates, which can be interpreted as evidence for the existence of variations in the distribution of stresses by size of inhomogeneities. The intensities of these variations estimated under Zhurkov's kinetic concept of fracture of solids are comparable to the stresses released by earthquakes. The statistical range of regional variations in the duration of the failure cycle is about two orders of magnitude; the durations are inversely proportional to the region-mean velocities of relative plate motions along the fault zones. This indicates that the durations of the failure cycle are mainly determined by the velocities of tectonic deformations. The significant scatter in the failure cycle duration values within regions testifies to the substantially heterogeneous intraregional distribution of the strain rates and strength, which is consistent with up-to-date notions of the hierarchical nature of a geological medium and mosaicity of the stress field.
\end{abstract}

Keywords: subduction zone, failure cycle duration, velocity of tectonic deformations

DOI: $10.1134 / \mathrm{S} 1069351319050124$

\section{INTRODUCTION}

The modern notions in the physics of failure rely on the mechanics of fracture and on the kinetics of a system of various-scale defects under the stress field. These notions contain a number of key parameters (e.g., material durability in the kinetic concept of fracture or critical crack density in mechanics) which determine the character of the failure process. These physical parameters for the seismogenic layer of the Earth can be estimated from earthquake catalogue data based on the notions on the structure of seismicity (Smirnov, 2003; Smirnov and Zavyalov, 1996).

A fundamental challenge to quantitative comparison of the results of seismic statistics with the predictions by the physical theories of material fracture is that the spatial domains in question have unequal sizes. A characteristic spatial scale in the physics of fracture is the source size of an earthquake. The estimates in seismic statistics refer to regions that include a lot of earthquake sources and, hence, are much in excess of the size of a single source. Transferring the statistical estimates obtained in natural conditions to the theoretical domain means extrapolation of these estimates on the scales of the spatial sizes. This extrapolation is correct only if it adequately accounts for the probable dependence of the parameters being estimated on the size of the domain (spatial scale) they are estimated for. The fairly reliable notions that have been developed to date on the fractal character of the spatial structure of seismicity provide a basis for transferring the estimates of seismic statistics to the spatial 
scales relevant for physics of fracture (Smirnov, 2003; Smirnov and Ponomarev, 2004).

In this paper, we analyze the parameters characterizing the duration of the failure cycle of the lithosphere based on a number of regional earthquake catalogues for territories with different tectonic regimes: subduction zones and continental shear transform fault zones. The duration of the failure cycle is understood as the mean time between two successive failures of a given domain of the medium. The failure cycle parameters are estimated based on the generalized Gutenberg-Richter relation for earthquake recurrence (Kossobokov and Mazhenkov, 1988) according to the previously developed scheme (Smirnov, 2003).

\section{FAILURE CYCLE PARAMETERS}

The Gutenberg-Richter frequency-magnitude relationship and fractal geometry of seismicity reflect the fundamental property of failure process-its selfsimilarity. The Gutenberg-Richter law expresses the statistics of the source sizes of the earthquakes: it shows how the total number of the earthquakes changes with their size. Fractal geometry determines the dependence of spatial density of the earthquakes on the size of region under study. The both regularities were integrated into one, subsequently come to be known as the generalized recurrence law (Kossobokov and Mazhkenov, 1988; 1994; Keilis-Borok et al., 1989) and, independently, by (Chelidze, 1990) or, in alternative terminology, as the unified scaling law for earthquakes (Bak et al., 2002):

$$
\log N=-b \log E+d \log L+\log T+\log B,
$$

where $N$ is the total number of earthquakes with energy $E$ that occurred in a region of size $L$ during time $T, b$ is the Gutenberg-Richter $b$-value, $d$ is fractal dimension of geometrical distribution of seismicity, and $B$ is the generalized seismic activity (here, the discussed law is recorded in the form accepted in seismology - with logarithms of the dimensional units implying that measurement units are specified separately). Later, the pioneering authors of the generalized frequency-magnitude relation of the earthquakes came to refer to it as a unified scaling law for the earthquakes (Kossobokov and Nekrasova, 2004; Nekrasova, 2008; Nekrasova and Kossobokov, 2005; 2006; Nekrasova et al., 2011; 2015; Kossobokov and Soloviev, 2018). We however adhere to the original name since, in our opinion, it more adequately reflects the matter of this law.

The generalized law combines the estimates of the frequency of the earthquakes obtained in the regions with different sizes for the events with different energies, which allows correct rescaling of the statistics obtained in the regions of different sizes. In particular, this law provides the basis for correctly extrapolating the representative statistical estimates of seismicity parameters obtained in the different volumes of the medium into the domain of the physically relevant spatial scales of the fracturing parts of the medium.

If we set in (1) the domain size $L$ equal to the source size $l$ of the earthquake and substitute the formula expressing the energy of the earthquake through its source size $E=E_{0} l^{a}$ (Sadovskii et al., 1983), we obtain the relation for the mean number of the earthquakes $N_{0}$ that occur in the domain with a size equal to their source size during time interval $T$, i.e. for the mean (over time $T$ ) number of fracture events of the domain of size $l$ : $\log N_{0}=-(a b-d) \log l+\log T-b \log E_{0}+\log B$. The mean frequency of occurrence of fracture events $v_{0}=N_{0} / T$ is in this case

$$
\mathrm{v}_{0}=\frac{B}{E_{0}^{b}} l^{-(a b-d)} .
$$

The duration of the failure cycle is estimated as the time interval inversely proportional to the mean recurrence rate of the earthquakes in a domain whose size is equal to the source size of these earthquakes. From (2) it follows that the dependence of failure cycle duration on size $l$ of the fracture domain (the source size) has the following form:

$$
\tau(l)=\tau_{0}\left(l / l_{0}\right)^{q} .
$$

Here, $\tau_{0}$ is the duration of the failure cycle for the domain $l_{0}$ (equal to the source size of an earthquake with magnitude $M_{0}$ ) and

$$
q=\alpha b-d,
$$

where $\alpha$ is determined by the relation between the magnitude and the source size of the earthquake:

$$
M=\alpha \log l+\beta .
$$

The estimates of parameters $b, d$, and $B$ contained in (2) and, accordingly, parameters $\tau_{0}$ and $q$ contained in (3) can be obtained for active seismic regions based on the data of earthquake catalogues. The procedure for their estimation is described in detail in (Smirnov, 2003).

The initial data for estimating the failure cycle duration $\tau$ are composed of the following statistics which are determined based on the earthquake catalogue data in a selected spatial cell: number $N$ of the events with magnitude $M \geq M_{\min }$ that occurred during time interval $T$ in a spatial domain of size $L$; $b$-value of the frequency-magnitude graph; and fractal dimension $d$ of the set of the hypocenters. Failure cycle duration $\tau$ is estimated for the events in the magnitude range $[M-\Delta M, M+\Delta M]$ which corresponds to the recurrence of fracture in the domain of the medium whose size lies in the range $\left[l_{1}, l_{2}\right]$. It is assumed that the seismicity obeys the recurrence law (1) in the range of completeness magnitudes $M \geq M_{c}$ and in a 
certain range of spatial scales $\left[L_{1}, L_{2}\right]$ known as a scaling interval.

The estimate of the failure cycle duration for the earthquakes with magnitude $M_{0}$ has the following form (Smirnov, 2003):

$$
\begin{gathered}
\tau_{0}=\frac{T}{N} \times 10^{-b M_{\min }} \\
\times\left(10^{b \Delta M}-10^{-b \Delta M}\right)^{-1}\left(\frac{10^{-\beta / \alpha}}{L}\right)^{-d} \times 10^{\frac{q}{\alpha} M_{0}} .
\end{gathered}
$$

The $q$ value is calculated by formula (4).

We note that formula (3) is similar to the classical Gutenberg-Richter frequency-magnitude relationship recast in terms of the source sizes of the events:

$$
\tau(l)=\tau_{0}\left(l / l_{0}\right)^{\alpha b},
$$

however, it not only takes into account the power-law dependence of the occurrence frequency of the earthquakes on their energy but also allows for the powerlaw dependence of the occurrence frequency of the earthquakes on the size of the seismogenic domain which is due to the fractal geometry of seismicity. Indeed, let us consider a situation when $n_{M}$ earthquakes with magnitude $M$ occurred in a region of size $L$ during time $T$. We denote the mean frequency of occurrence of the earthquakes in this domain by $v(L)=n_{M} / T$. If earthquakes were uniformly distributed in space, the mean frequency of their occurrence in a domain of size $l$ would be fewer by a factor of $(L / l)^{3}: v(l)=v(L)(l / L)^{3}$. However, the spatial distribution of the earthquakes is nonuniform. If we assume that the geometry of seismicity is fractal, then instead of factor $(l / L)^{3}$ in the calculation of $v(l)$ we should use $(l / L)^{d}$ where fractal dimension (or, more accurately, cluster dimension) is $d$. It is this "fractal correction" that is contained in formula (3), thus making it possible to calculate the mean frequency of occurrence of the earthquakes in a domain of size $l$ based on the mean frequency of occurrence of the earthquakes in a larger domain of size $L$ where the number of the events is sufficient for obtaining the statistically representative estimates.

In accordance with (3), we consider parameter $q$ and quantity $\tau_{0}$ as physical parameters of the failure cycle. By analogy with the Gutenberg-Richter $b$-value, we refer to parameter $q$ as the $q$-value (Smirnov, 2003).

The parameters of the failure cycle are estimated within spherical spatial cells with radius $R_{0}$ that are moved in space with step $\Delta D$ in the horizontal directions and with step $\Delta Z$ along the depth. Prior to the calculations, the earthquake catalogue is declustered to remove the aftershocks. The aftershocks were identified based on the author's software (Smirnov, 1997a; 2009) implementing the Molchan-Dmitrieva algorithm (Molchan and Dmitrieva, 1991; 1993; Molchan et al., 1996).

\section{ERRORS OF THE FAILURE CYCLE PARAMETERS ESTIMATES}

The estimate of $\tau_{0}$ is calculated as an inverse to the mean frequency of occurrence $n_{M l}$ of the earthquakes of selected magnitude $\tilde{M}$ in the spatial domain of size $\tilde{l}$ corresponding to the source size of the event wjth magnitude $\tilde{M}$. According to (6), the formula for $b$ contains three product terms (separated in braces):

$$
n_{M l}=\left\{\frac{N}{T}\right\}\left\{10^{-b \tilde{M}} 10^{b M_{\min }}\left(10^{b \Delta M}-10^{-b \Delta M}\right)\right\}\left\{\left(\frac{\tilde{l}}{L}\right)^{d}\right\},
$$

where $\tilde{l}$ and $\tilde{M}$ are linked by relation (5).

The first product term in formula (8) is determined by the frequency of occurrence of all the earthquakes (within the entire magnitude range $\left[M_{\min }, \infty\right]$ ) in a domain with size $L$ during time $T$. The second multiplier expresses the conversion of this frequency to the selected magnitude or, to be more accurate, to the magnitude range $[\tilde{M}-\Delta M, \tilde{M}+\Delta M]$. The third term renders downscaling of the estimate from the domain of size $L$ to the source area $\tilde{l}$. Accordingly, the error of the estimate of quantity $\log n_{M l}$ and the error of $\log \tau_{0}$ which is equal to the latter have three consituents:

$$
\begin{gathered}
\Delta \log \left(\frac{N}{T}\right) ; \Delta b\left(\tilde{M}-M_{\min }\right)+\Delta \log \left(10^{b \Delta M}-10^{-b \Delta M}\right) ; \\
\Delta d \log \left(\frac{\tilde{l}}{L}\right) .
\end{gathered}
$$

The random component of $\Delta \log \tau_{0}$ is due to the estimation errors of $N, b$ and $d$ over a finite sample corresponding to the considered spatiotemporal window whereas the systematic error, identical for all windows, is associated with errors of coefficients $\alpha$ and $\beta$ (the other parameters $T, \tilde{M}, M_{\min }, \Delta M$, and $L$ are specified accurately).

The error of $\log \tau_{0}$ at the points of the numerical grid is estimated by the following formula

$$
\Delta \log \tau_{0}=\sqrt{\frac{1}{(\ln 10)^{2} N}+\left(\Delta b\left(\tilde{M}-M_{\min }+\Delta M\right)\right)^{2}+\left(\Delta d \log ((L / \tilde{l}))^{2}\right.}
$$


The error of $q$-value is calculated in a standard way based on the errors of parameters $b$ and $d$ :

$$
\Delta q=\sqrt[2]{(\alpha \Delta b)^{2}+\Delta d^{2}}
$$

\section{ESTIMATES OF FAILURE CYCLE PARAMETERS}

The first statistical estimates for the duration of the failure cycle were obtained based on (1) in (KeilisBorok et al., 1989) for several regions which were selected in accordance with the set of the earthquake catalogues used in the cited study. Below, we present the results of estimating the failure cycle parameters and their spatial distributions in the subduction zones with Kamchatka and Japan as the examples and in the shear zones on the transform plate boundaries with the evidence from the regions of San-Andreas and North Anatolian faults as well as the Zagros zone, Iran.

Kamchatka. The earthquake catalogue is presented on the website of the Kamchatka Branch of the Geophysical Survey of the Russian Academy of Sciences ${ }^{1}$ (the version without volcanic earthquakes). The Kamchatka catalogue covers the period since 1962. During this more than 50-year interval, the catalogue underwent substantial changes associated with upgrading the network configuration and advancing the methods for processing the initial data and calculating the parameters of the earthquakes (Gordeev et al., 2011; 2013). The earthquake catalogue contains information about origin times, epicentral coordinates and depths, and energy class of the events which is linked with the energy of the earthquake by the relation $K=\log E$ with $E$ expressed in $\mathrm{J}^{2}$

The completeness of the Kamchatka catalogue was tested by two methods: with the use of Z-map program package ${ }^{3}$ and with the authors' software (Smirnov, 1997a; 2009) implementing the technique of (Pisarenko, 1989; Sadovskii and Pisarenko, 1991). The results are shown in Figs. 1 and 2. The both methods yielded mutually consistent estimates for the changes in catalogue's completeness with time and for the distribution in spatiotemporal domains.

The estimates of the class of completeness show that it varies from 6.5 to 9.5 depending on time and location and exceeds the level $K_{c}=9.5$ only at separate time instants in the extreme southern part of the region. With this taken into account, in order that the working catalogue be uniform throughout the entire period starting from 1964, only the events with $K \geq 9.5$ were included in this catalogue.

\footnotetext{
${ }_{1}^{1}$ http://sdis.emsd.ru/info/earthquakes/catalogue.php

2 This relation should be considered as statistical rather than physical because energy class pertains to the type of magnitude estimates (Abubakirov et al., 2018).

${ }^{3} \mathrm{http}: / /$ www.geociencias.unam.mx/ ramon/ZMAP/intro.html
}

The results of estimation of $\tau_{0}$ and $q$ from the catalogue of the Kamchatka earthquakes are illustrated in Fig. 3; the $\tau_{0}$ value corresponds to energy class 16 . In the calculation formulas (6) and (9), magnitude $M$ is replaced by energy class $K$, parametets in (5) have the values $\alpha=3, \beta=11$ according to (Sadovskii et al., 1983). The $\Delta K$ value was selected based on the condition $l_{2} / l_{1}=3$ (Smirov, 2003) which corresponds to the neighboring hierarchical levels of lithospheric inhomogeneities according to (Sadovskii and Pisarenko, 1991 ) and was 0.72 of energy class unit.

The upper bound of the scaling interval for estimating the fractal dimension in moving spatial windows was $100 \mathrm{~km}$ which allowed us to separate the estimates of failure cycle parameters into two intervals by depth. The map constructed for the whole (undifferentiated) seismogenic layer is presented in Fig. 3a whereas Figs. 3b and $3 \mathrm{c}$ show the results of the calculations separated into two layers by depth.

The interquartile range of the estimates of failure cycle duration over the entire region is 400 to 2000 years. In Fig. 3 it can be seen that shortest duration of the failure cycle is confined to the main seismofocal region in the subduction zone. In its upper part stretching along the Kamchatka coast, the duration of the failure cycle is a few hundred years which, as noted in (Smirnov, 2003), agrees with the estimates of seismic cycle duration obtained by S.A. Fedotov (1968) (with the adjustment for the procedure used by Fedotov). As the depth of the seismogenic region increases along the subducting oceanic plate, the duration of the cycle grows approximately by the order of magnitude. Beyond the main seismofocal zone, the duration of the failure cycle is almost three orders of magnitude as large as within the zone.

The $q$-value distribution is not stuck to the main structural features of the region. In Fig. 3 it can be seen that almost throughout the entire territory, the $q$-value is close to zero within its mean estimation error: the region of these values is shown in Fig. 3 in yellow.

The anomalous values of failure cycle parameters at the extreme edges of the selected frame are probably due to the heterogeneity of data on the periphery of the region covered by the seismic catalogue.

For elucidating on the presence of the statistically significant spatial variations in the parameters of the failure cycle, we carried out the analysis of variance based on the data for the entire region. We compared the serial variance of the set of the estimates obtained from the set of the selected spherical cells with the mean statistical error of individual estimates in each cell obtained by formulas (9) and (9a). The results are presented in Table 1.

From the last right column of Table 1 it can be seen that the serial variance for the both parameters is greater than the statistical error of estimates testifying 


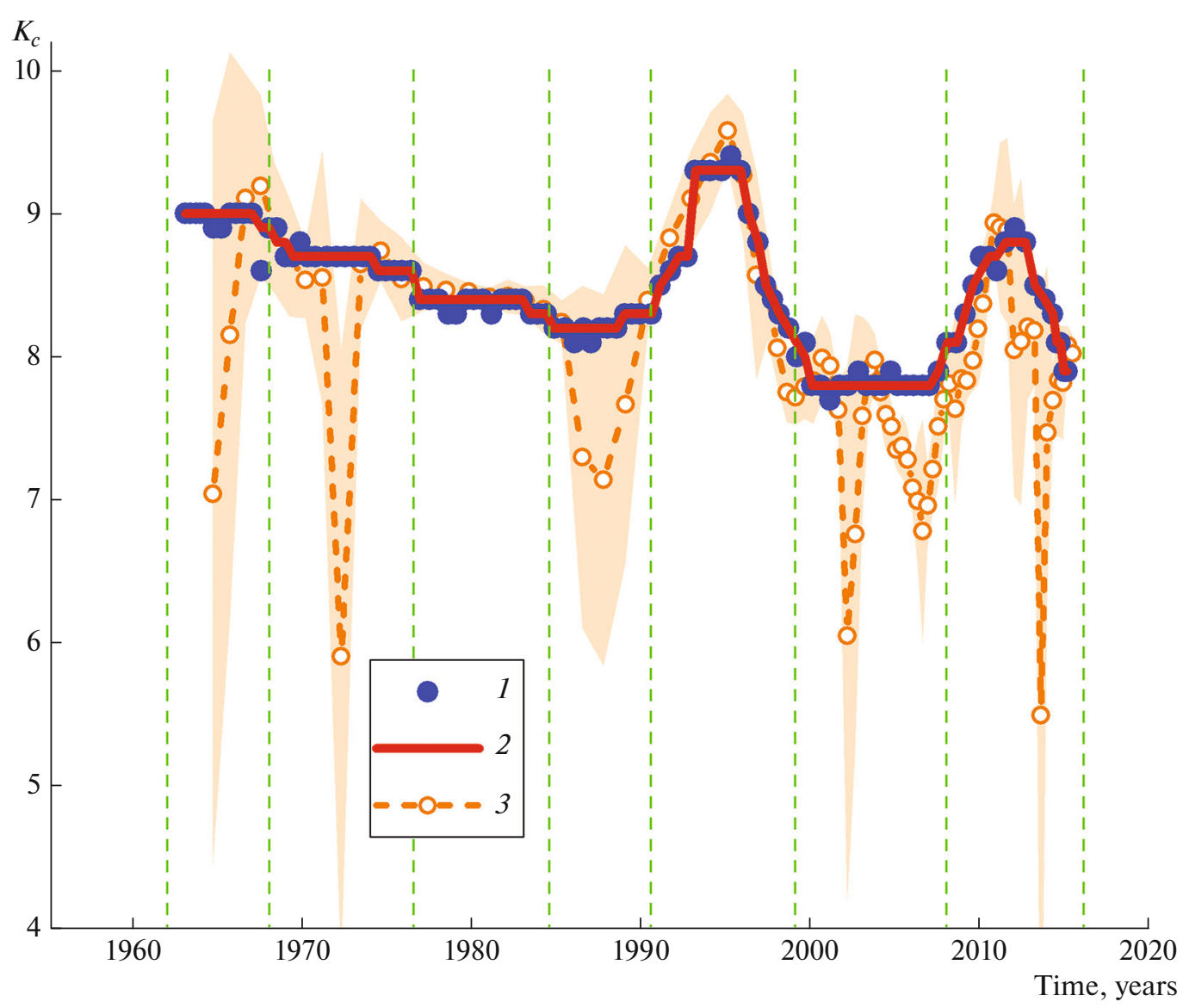

Fig. 1. Changes of class of completeness $K_{c}$ for Kamchatka catalogue with time based on data over entire region. 1, 2, authors' original software: 1 , initial estimates; 2 , averaged curve; 3, Z-map package, confidence intervals are shown by filling. Vertical dashed lines show selected intervals of seismic network development.

to the presence of the significant spatial variations in the parameters of the failure cycle.

Japan. As the initial data for this region we used the records from the International Seismological Center (ISC ${ }^{4}$ ) earthquake catalogue contributed by the Japan Meteorological Agency (JMA). The catalogue provides local magnitudes. The orthogonal regression of the $M_{W}$ values presented in the Harvard Centroid Moment Tensor Catalog and magnitude $M$ of JMA catalogue selected for this study gives the relation

$$
M_{W}=(0.98 \pm 0.02) M+(0.24 \pm 1),
$$

which is close to the relation $M=M_{W}$. Considering the relationship $\log l(\mathrm{~km})=\frac{1}{3} \log M_{0}(H \cdot \mathrm{m})-5.07$ (Gusev and Mel'nikova, 1990), we obtain $\alpha=2.04$ and $\beta=3.91$ for the parameters in (5).

The analysis of the completeness magnitude has shown that starting from 1981, all the events with mag-

\footnotetext{
${ }^{4} \mathrm{ftp}: / /$ isc-mirror.iris.washington.edu/pub/
}

nitudes $M \geq 3.3$ throughout the entire territory are reported completely.

Figure 4 shows the distribution maps of parameters $q$ and $\tau_{0}$ for the entire seismogenic layer. Based on the JMA catalogue, it is also possible to estimate the failure cycle parameters separately for two layers along the depth: above $50 \mathrm{~km}$ and in the depth interval from 50 to $100 \mathrm{~km}$. The respective maps are shown in Fig. 5.

Table 2 presents the results of the analysis of variance for the obtained estimates of the failure cycle parameters. By examining these data we can compare the degree of spatial variability of these estimates (in

Table 1. Results of analysis of variance for failure cycle duration estimates based on Kamchatka catalogue

\begin{tabular}{l|c|c|c}
\hline Parameter & $\begin{array}{c}\text { Square root } \\
\text { of serial variance } \sigma_{s}\end{array}$ & $\begin{array}{c}\text { Mean error } \\
\text { of individual } \\
\text { estimates } \sigma_{0}\end{array}$ & $R=\frac{\sigma_{s}}{\sigma_{0}}$ \\
\hline$q$ & 0.43 & 0.25 & 1.7 \\
$\log \tau_{0}$ (year) & 0.82 & 0.34 & 2.4 \\
\hline
\end{tabular}




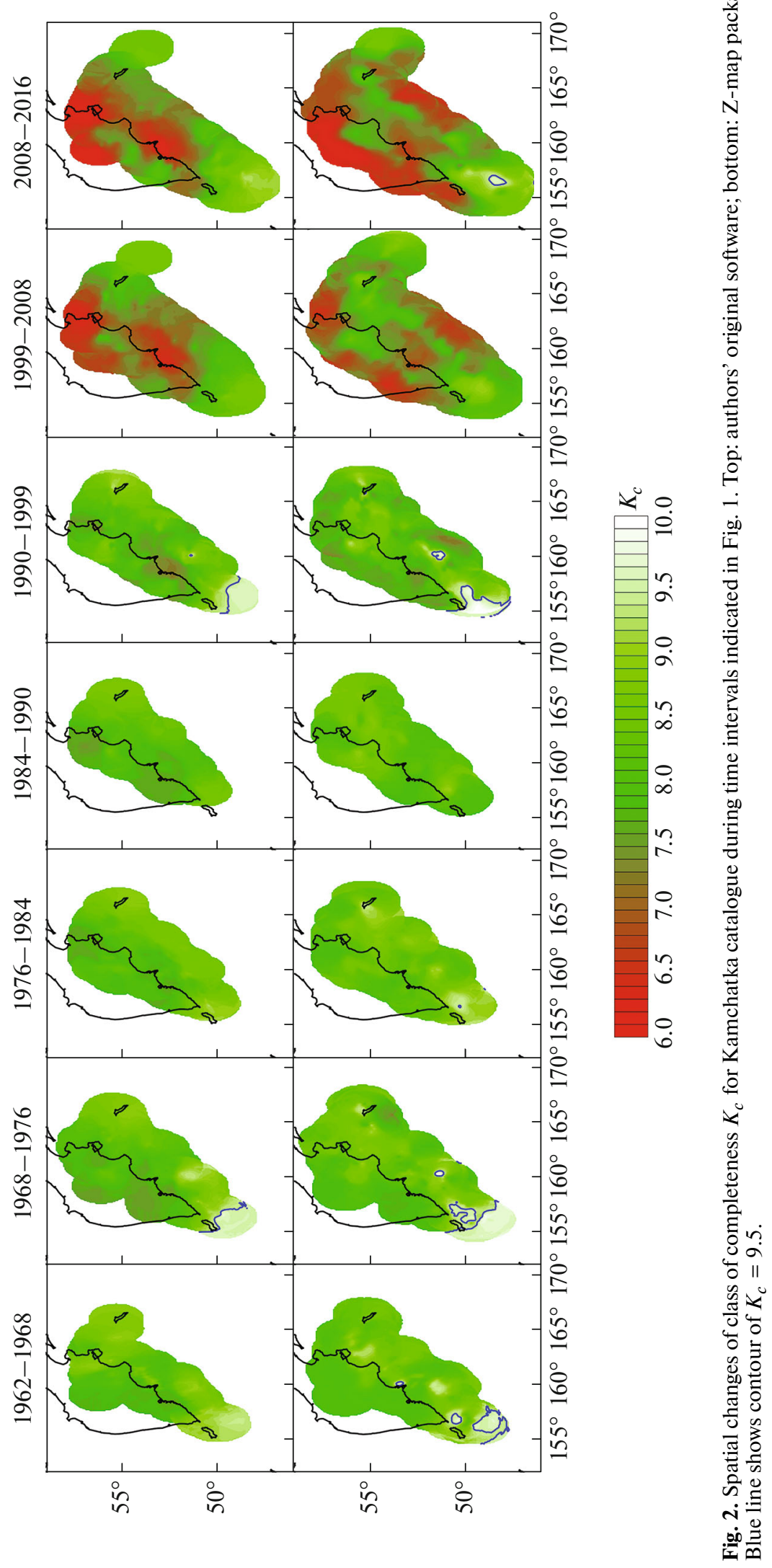



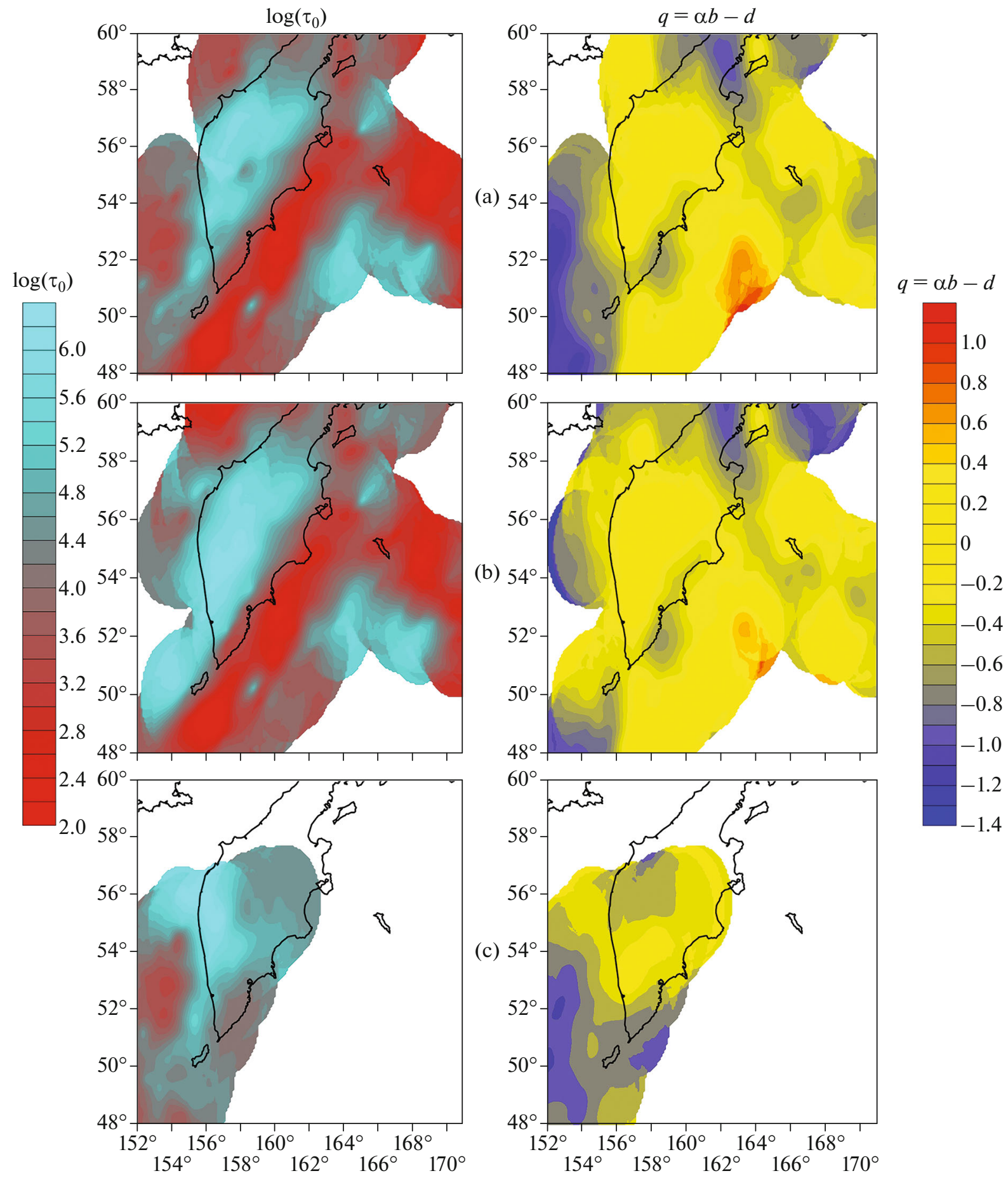

Fig. 3. Failure cycle duration $\tau_{0}$ (in years, for $K_{0}=16$ ) and $q$-value, based on regional earthquake catalogue for Kamchatka. Areas with zero (within error) $q$-values are shown in yellow: (a) for entire seismogenic layer; (b) for depths above $150 \mathrm{~km}$; (c) for depth below $150 \mathrm{~km}$.

terms of the root of serial variance $\sigma_{S}$ ) with the mean statistical error of the estimate $\sigma_{0}$.

The mean $q$-value for Japan is -0.07 . With the presented variance values taken into account, it can be assumed that the mean $q$ is zero, i.e., on average, the duration of the failure cycle does not depend on the size of the fracture area. Moreover, the presented variance estimates show that the spatial variability of the parameters is 1.6 times as large as the statistical error which means that the spatial variations of the considered parameters are real (statistically significant). 


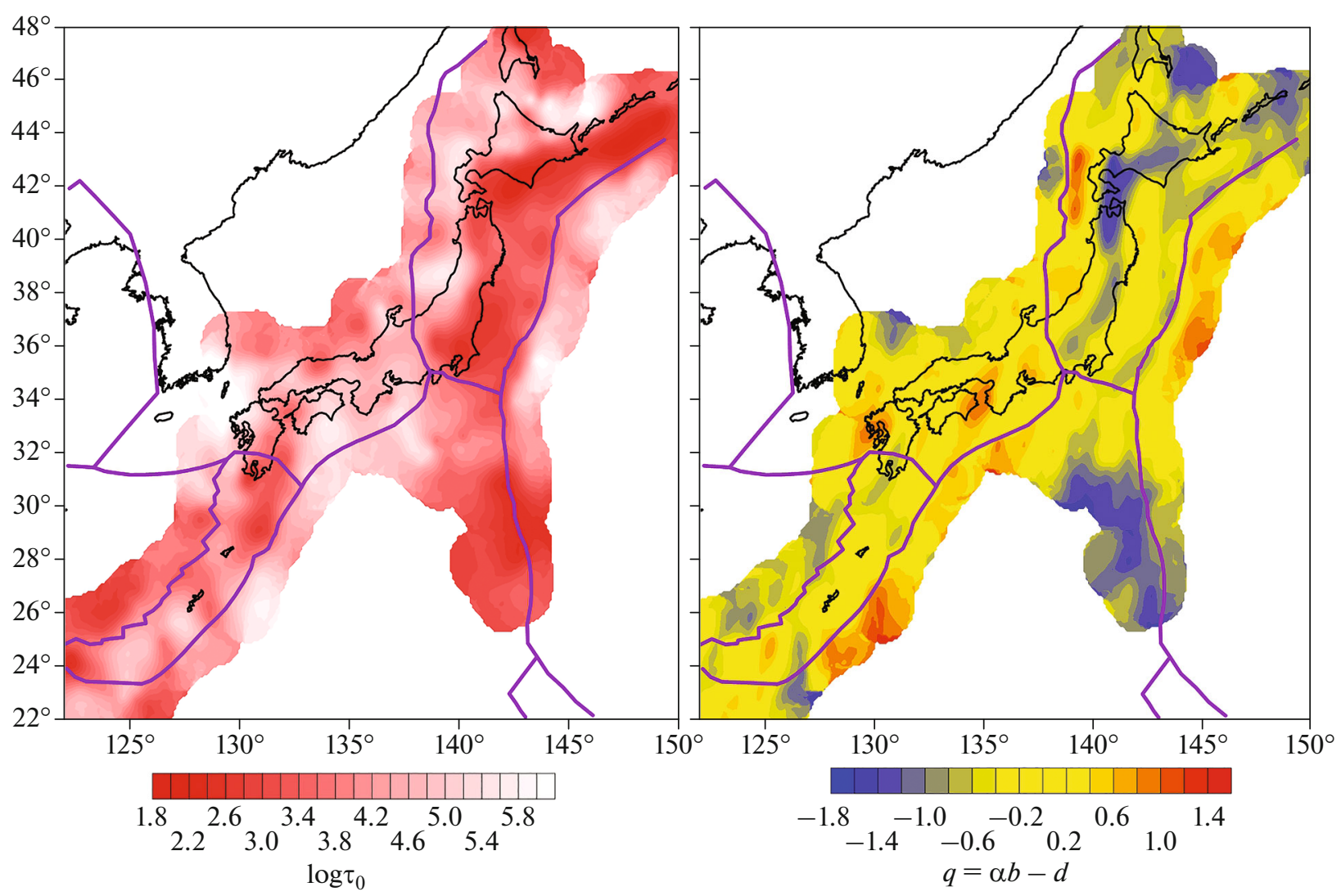

Fig. 4. Failure cycle duration $\tau_{0}$ (in years, for $M_{0}=7.5$ ) and $q$-value, based on regional earthquake catalogue for Japan. Areas with zero (within error) $q$-values are shown in yellow.

The spatial distribution of the $q$-value has the areas of positive and negative values. The positive $q$-values are confined to the plate boundaries in the oceanic part of the subduction zone. The negative are $q$-values are observed behind the deep oceanic trenches; they fall in the contact zone of the oceanic and continental plates and on the subsiding part of the plate (this is most clearly pronounced in Fig. 5 for the depth from 50 to $100 \mathrm{~km}$ ).

The nature of this structure of $q$-value distribution is unclear. However, it could hypothetically be related to the difference in the characteristic stresses: in the region of positive $q$-values, the plate undergoes bending and its upper part may accommodate tensile stresses. We note that based on the global earthquake catalogue data, positive $q$-values were previously

Table 2. Results of analysis of variance for failure cycle duration estimates based on catalogue for Japan

\begin{tabular}{l|c|c|c}
\hline Parameter & $\begin{array}{c}\text { Square root } \\
\text { of serial variance } \sigma_{s}\end{array}$ & $\begin{array}{c}\text { Mean error } \\
\text { of individual } \\
\text { estimates } \sigma_{0}\end{array}$ & $R=\frac{\sigma_{s}}{\sigma_{0}}$ \\
\hline$q$ & 0.63 & 0.4 & 1.6 \\
$\log \tau_{0}$ (year) & 0.95 & 0.6 & 1.6 \\
\hline
\end{tabular}

established for the rift zones-the characteristic regions of extension (Smirnov, 2003). The region of negative $q$-values for Japan corresponds to the zone of compression.

The interquartile range of the estimates of failure cycle duration over the entire region is 900 to 5700 years.

Northern California. The initial data for this region were taken from the catalogue of the Northern California Earthquake Data Center. ${ }^{5}$ The main magnitude type in this catalogue is the magnitude estimated from the duration of coda waves-the coda magnitude, $M_{d}$. However, this magnitude becomes saturated at 4.5 and higher. Therefore, the authors of the catalogue provide the Preferred Magnitude field for the composite scale which they recommend to use. In this scale, the relatively strong events with magnitudes above 4 are provided with the magnitude values in the scales different than $M_{d}$ (mainly, the Richter magnitudes $M_{L}$ ). Judging by the frequency-magnitude graph, this composite scale can be accepted as sufficiently uniform for solving our problem.

The orthogonal regression of the $M_{W}$ values from the Harvard Centroid Moment Tensor Catalog and

$\overline{5^{5} \text { http://quake.geo.berkeley.edu/ncedc/catalog-search.html }}$ 

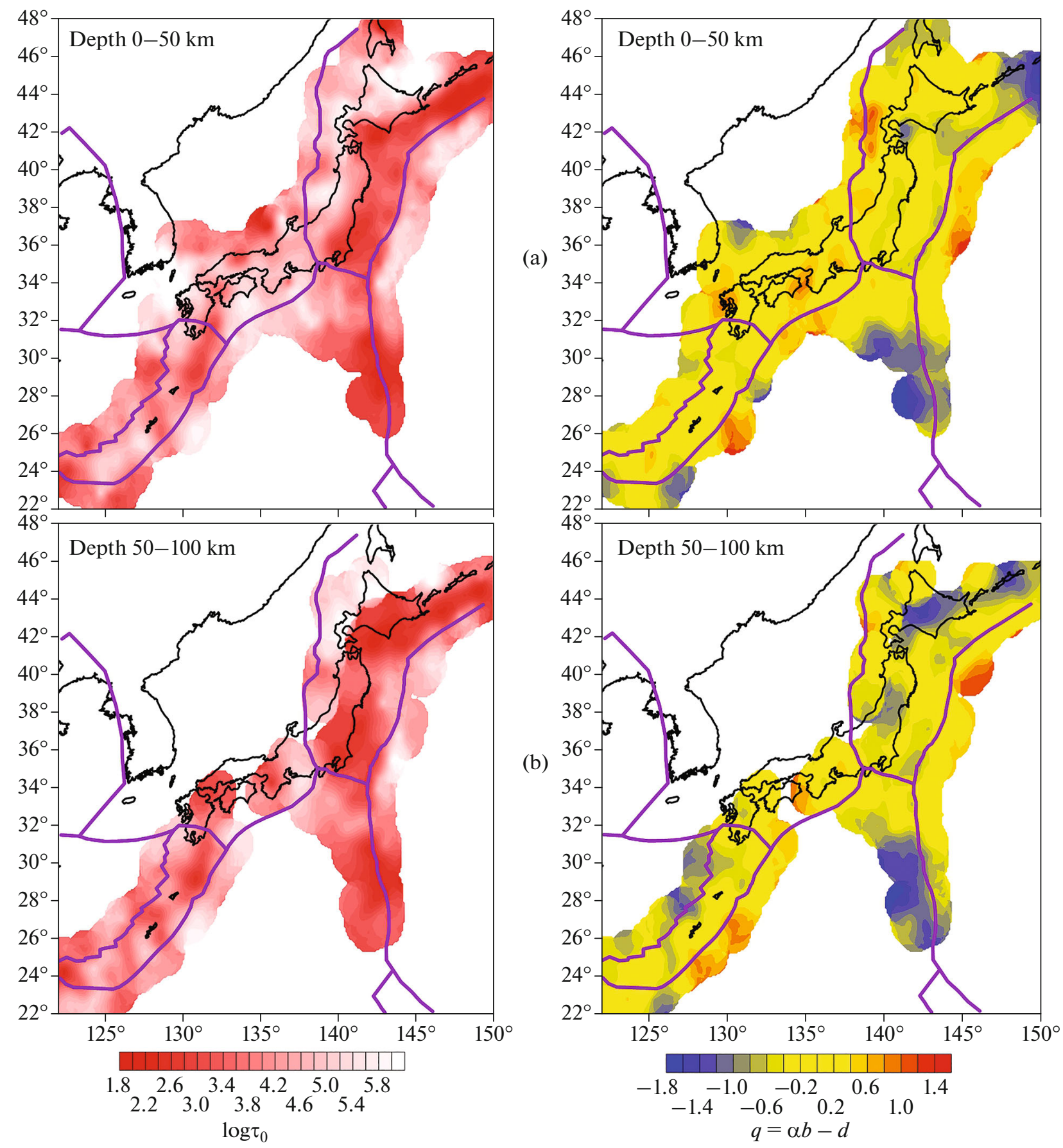

Fig. 5. Failure cycle duration $\tau_{0}$ (in years, for $M_{0}=7.5$ ) and $q$-value, based on regional earthquake catalogue for Japan for depths (a) $0-50$ and (b) $50-100 \mathrm{~km}$. Areas with zero (within error) $q$-values are shown in yellow.

"composite" magnitude $M$ from the Northern California Catalog gives the following fit:

$$
M_{W}=(0.99 \pm 0.1) M+(0.19 \pm 0.5) .
$$

Just as in the case of the catalogue for Japan, this is close to the relation $M=M_{W}$. The values of parameters in (5) are $\alpha=2.02$ and $\beta=3.93$. Starting from
1978, the magnitude value of 2.3 can be accepted as the magnitude of completeness for the entire territory.

Figure 6 shows the distribution maps of parameters $q$ and $\tau_{0}$. The results of the analysis of variance are presented in Table 3.

The region-mean $q$-value $q=0.2$ is within the mean estimation error. In Fig. $6 b$ it can be seen that 
(a)

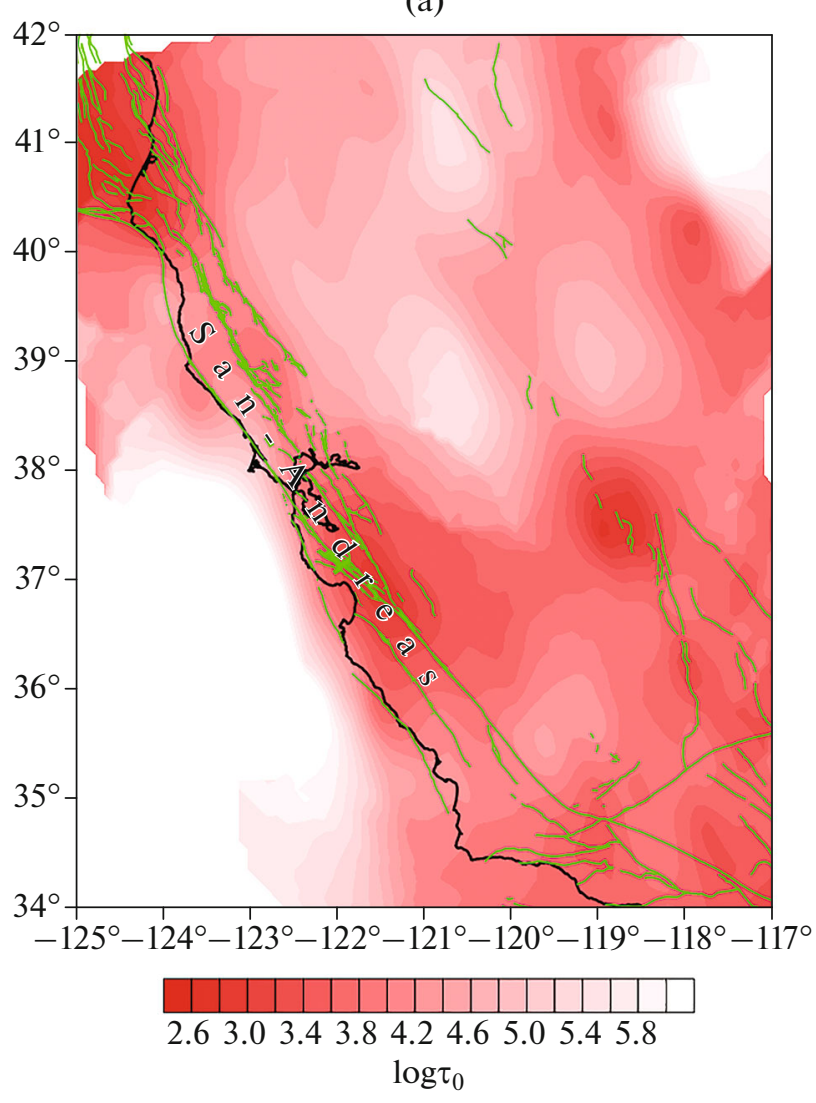

(b)

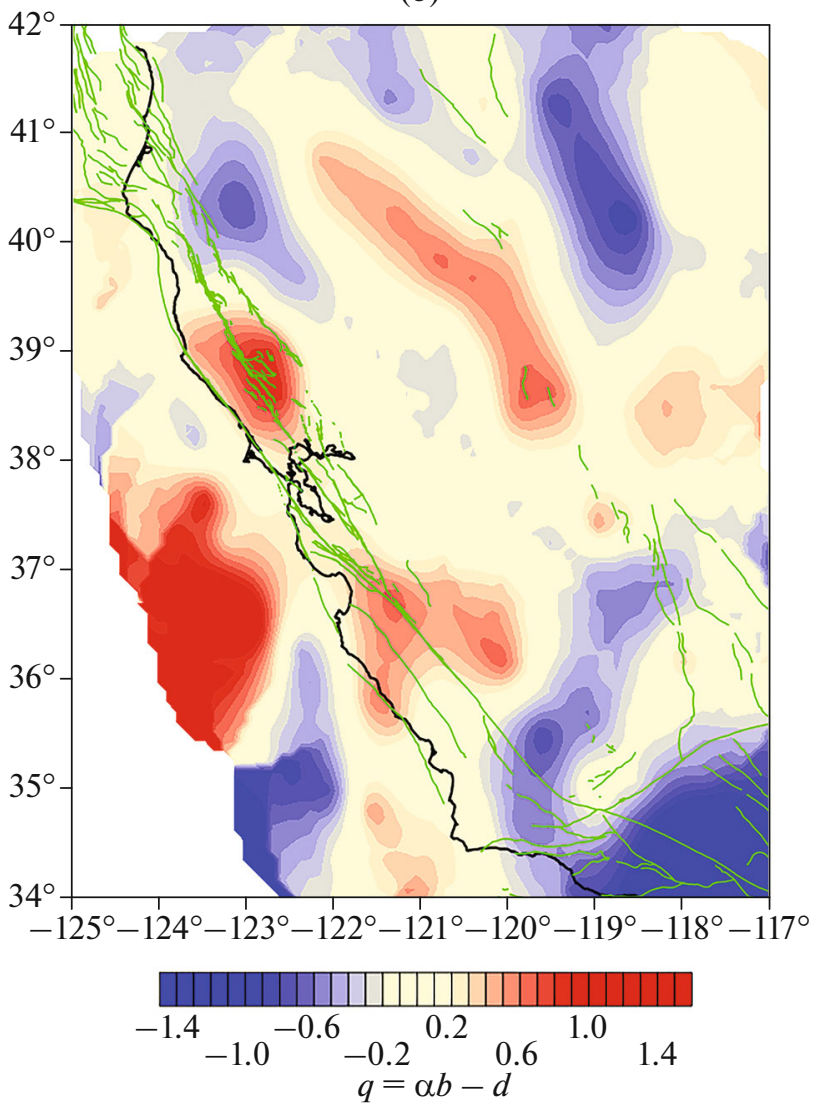

Fig. 6. Failure cycle duration $\tau_{0}$ (in years, for $M_{0}=7.5$ ) and $q$-value, based on regional earthquake catalogue for Northern California. In Fig. 6b, areas with zero (within error) $q$-values are shown in yellow.

anomalously high (by absolute value) $q$ fall on the edge of the region where the catalogue data are least reliable; estimation error of $q$ in these zones is largest: the relative error reaches $100 \%$. As for the rest, in most of the region the $q$ values are close to zero. In Fig. 6b, the values $q=0 \pm \sigma_{0}$ are shown un yellow.

The interquartile range of the estimates of the failure cycle duration over the entire region is $3500-7500$ years.

The analysis of variance (Table 3 ) shows that the intraregional variations in the parameters of the failure cycle are statistically significant.

Table 3. Results of analysis of variance for failure cycle duration estimates based on Northern California Catalog

\begin{tabular}{l|c|c|c}
\hline Parameter & $\begin{array}{c}\text { Square root } \\
\text { of serial variance } \sigma_{s}\end{array}$ & $\begin{array}{c}\text { Mean error } \\
\text { of individual } \\
\text { estimates } \sigma_{0}\end{array}$ & $R=\frac{\sigma_{s}}{\sigma_{0}}$ \\
\hline$q$ & 0.56 & 0.2 & 2.8 \\
$\log \tau_{0}$ (year) & 0.98 & 0.4 & 2.4 \\
\hline
\end{tabular}

Greece. As the initial data we used the catalogue of the National Observatory of Athens, Institute of Geodynamics. ${ }^{6}$ The catalogue provides local magnitudes $M_{L}$. The comparison of the regional catalogue data for Greece with ISC and Harvard Centroid Moment Tensor catalogues shows that the magnitude scale of the regional catalogue is uniform in time.

According to (Papazachos and Papazachou, 1997), local magnitudes $M_{L}$ are linked with the moment magnitude $M_{w}$ by the following relation:

$$
\begin{gathered}
M_{W}=(0.97 \pm 0.02) M_{L}+(0.58 \pm 0.09) \\
\text { for } 3.0 \leq M_{L} \leq 6.0,
\end{gathered}
$$

which gives $\alpha=2.0$ and $\beta=3.28$ for the parameters in (5).

The maps of the failure cycle parameters are shown in Fig. 7. They are superimposed on the tectonic map of the region presented in (Papazachos and Papazachou, 1997).

\footnotetext{
${ }^{6}$ http://www.gein.noa.gr/en/

http://www.gein.noa.gr/services/cat.html
} 
Remarkably, the area of shortest failure cycle durations is spatially concurrent with the western termination of the North Anatolian fault and is stretched further westwards up to the Cephalonia Transform Fault (CTF) which connects the zone of the continental collision in the north with the subduction zone in the south. This region is dominated by shear seismotectonic deformations (Papazachos and Papazachou, 1997). Figure $7 \mathrm{~b}$ shows that in the discussed region, the $q$-values are close to zero: their deviations from zero are within the range of statistical errors (shown in yellow in Fig. 7b).

The interquartile range of estimates of failure cycle duration over the entire region is 6300 to 12500 years.

Iran. The regional catalogue for Iran was compiled by the International Institute of Earthquake Engineering and Seismology, Iran (IIEES). In order that the working catalogue for calculating the failure cycle parameters be uniform, the selection threshold in magnitude was accepted at 4.5. For the period before 1995, significant part of the events in the Iran catalogue is presented by the NEIC catalog data. Testing the uniformity of the catalogue has shown that the data from the Iranian network that replace and complement the NEIC data for the subsequent years correspond by their magnitude scale to the NEIC catalog. Based on this, for the parameters of the relation (5) linking the magnitude with the source size we used the estimates obtained in (Smirnov, 2003): $\alpha=1.11$ and $\beta=4.73$.

The calculation results for the parameters of failure cycle are illustrated in Fig. 8 (the duration of the failure cycle is referred to $m_{b}=7$ ). In the figure it can be seen that the characteristic $q$-values of the most active part of Iran - the Zagros shear zone-are close to zero.

The interquartile range of the estimates of seismic cycle duration over the entire region is 5000 to 11600 years; the highest activity (minimum duration values) is associated with the Zagros shear zone whereas the maximum activity corresponds to the junction of this zone in the south with the zone of compression.

\section{DISCUSSION $q$-Value}

From formulas (2) and (3) it follows that parameter $q=\alpha b-d$ characterizes the dependence of the failure cycle duration on the scale of fracture (the size of the fractured area). At $q=0$, fracture is uniformly distributed over the scales: the probability of a defect (an inhomogeneity of the medium) to fracture foes not depend on its size. If $q<0$, fracture process is more intense on the larger scales, and if $q>0$ it is more intense on the smaller scales.

In the fracture model of different-scale defects of the medium with power-law size distribution (in particular, for fractal geometry of a system of defects) at $q=0$, the Gutenberg-Richter law is exclusively deter- mined by the size distribution of the defects. Indeed, in this model, the number $d N$ of the earthquakes with energy $E$ and source size $l$ that occurred within a certain domain of the medium during a fixed time interval is determined by the product of the number $n$ of defects having a respective size and the probability $p$ of their fracture:

$$
d N(E)=n(l) p(l) d l
$$

where seismic energy and source size are linked by relation (5); in terms of (10), this relation has the form $\log E=\alpha \log l+\beta$. The probability of a defect with size $l$ to fracture during a certain time interval is estimated by the frequency $v$ of fracture of these defects which is inversely proportional to the duration of the failure cycle (3): $v(l)=v_{0}\left(l / l_{0}\right)^{-q}$. If $q=0$, then $p(l) \propto v(l)=$ const and $d N(E) \propto n(l) d l$, i.e. the distribution of the earthquakes by energies is solely determined by the distribution of the defects by sizes. If, furthermore, the size distribution of the defects is power-law $n(l) \propto l^{d}$, we obtain $d N(E) \propto l^{d} d l$ or, if we convert the size to energy according to (5),

$$
d N(E) \propto E^{-d / \alpha} d E .
$$

Comparing (11) to the Gutenberg-Richter relation $d N(E) \propto E^{-b} d E$, we find that $b=d / \alpha$ or

$$
d=\alpha b .
$$

Relationship (12), as reflecting the structure of inhomogeneities of the lithosphere in the energy structure of seismicity, has been first proposed in the explicit form by Aki (1981) and subsequently discussed by many authors in numerous works with regard to background seismicity (King, 1983; Grigoryan, 1988; Turkott, 1992; Smirnov, 1995; 1997b; 2003; Smirov and Ispolinova, 1995; Bak et al., 2002; Corral, 2005). In a broader sense based on multifractal measures, the relationship between the spatial and temporal structure of seismicity was considered in (Stakhovsky, 2001; 2004a; 2004b) and generalized in (Stakhovsky, 2017).

In (Smirnov, 1995), for explaining the relationship between self-similarity exponents of seismic process and, in particular, relation (12), the author suggested a postulative model which formalized the notions established by that time (Aki, 1981; Grigoryan, 1988; Ulomov, 1987; 1993). The idea of this model is that for a source area of an occurred earthquake, the ban is introduced (postulated) for the occurrence of the other earthquakes of the same magnitude during a certain time. In the theory of active media, this time is named a refractory period and is associated with the depletion of the resource for the next event. Relation (12) is obtained under the assumption that the refractory period of an earthquake is independent on magnitude and that the seismogenic region is geometrically fractal. This approach is, in fact, equivalent to the notion 

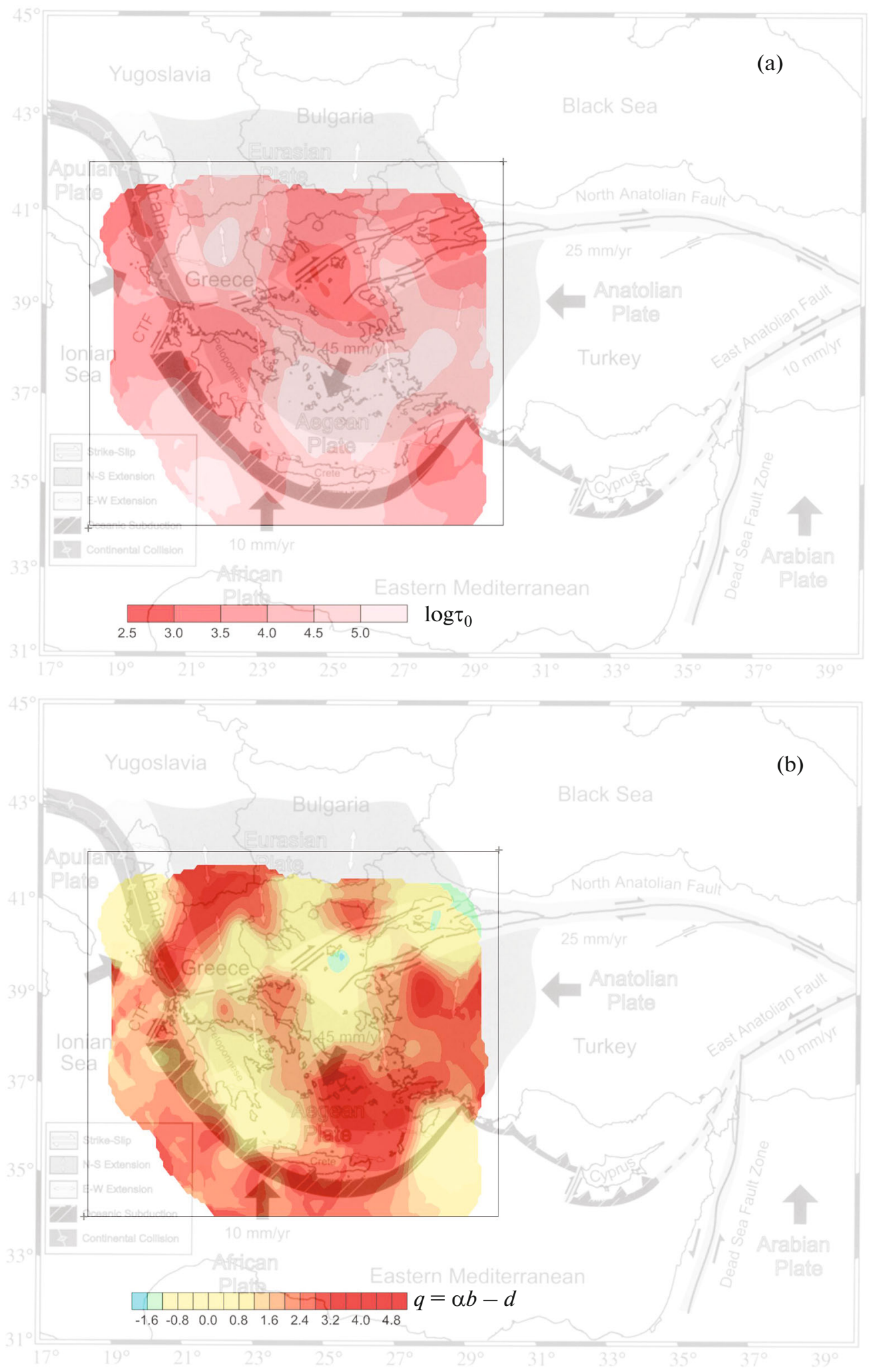

Fig. 7. Failure cycle duration $\tau_{0}$ (in years, for $M_{0}=7.2$ ) and $q$-value, based on regional earthquake catalogue for Greece. In Fig. $7 \mathrm{~b}$, areas with zero (within error) $q$-values are shown in yellow. 
(a)

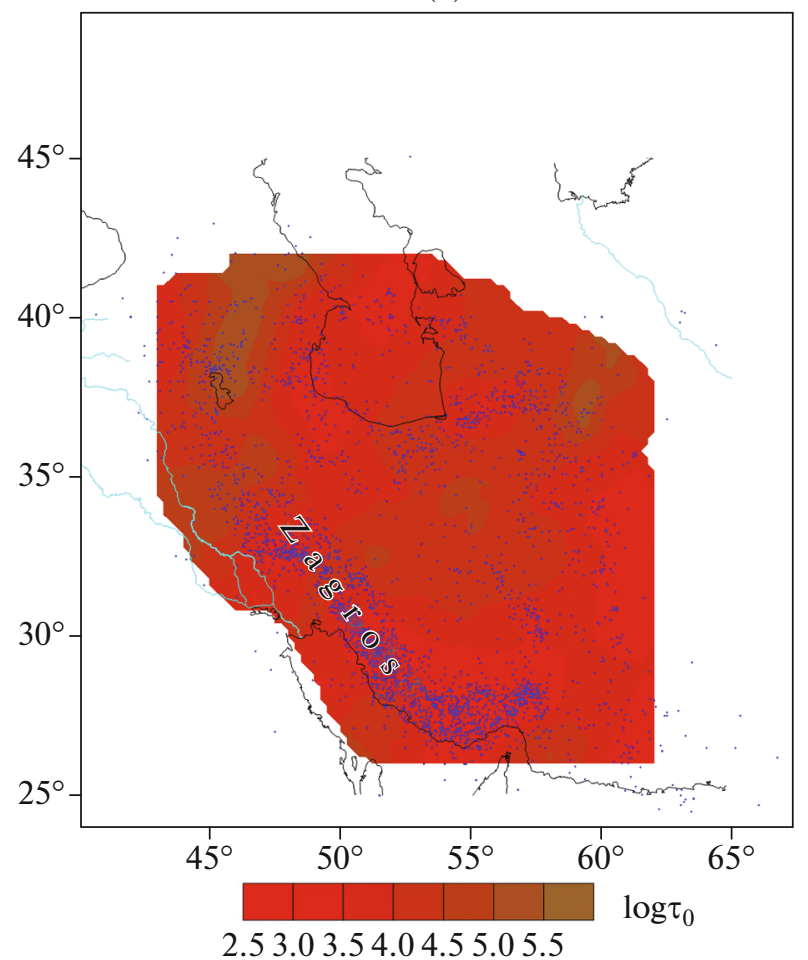

(b)

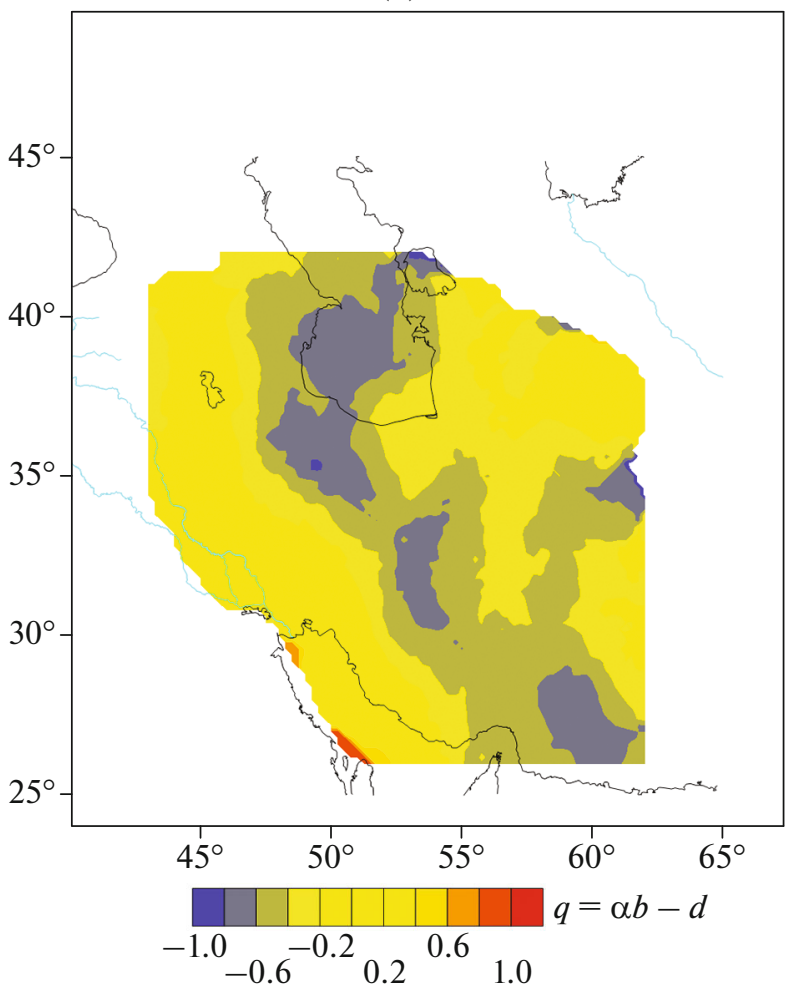

Fig. 8. Failure cycle duration $\tau_{0}$ (in years, for $m_{b}=7.0$ ) and $q$-value, based on regional earthquake catalogue for Iran. In Fig. 8a, dots indicate earthquake epicenters; position of Zagros zone is shown. In Fig. 8b, areas with zero (within error) $q$-values are shown in yellow.

that the distance between earthquake sources is proportional to their sizes (Smirnov and Ponomarev, 2004).

Parameter $q=\alpha b-d$ characterizes the deviation of a seismic process from relation (12). It shows the difference in the scalings (the exponents) of the statistical distributions for the source sizes of the earthquakes (exponent $\alpha b$ ) and for the distances between the earthquakes (exponent $d$ ). If these distributions agree, relation (12) is valid and, as noted above, the Gutenberg-Richter frequency-magnitude relationship is exclusively determined by the geometrical structure of the inhomogeneities of the medium.

Mean $q$-Value. The obtained estimates for the $q$-value show that on average, this parameter is close to zero. This main result is consistent with the estimates determined on both the global and regional level (KeilisBorok et al., 1989; Smirnov, 2003; Kossobokov and Nekrasova, 2004; Nekrasova and Kossobokov, 2006; Nekrasova, 2008; Nekrasova et al., 2011; 2015). Thus, the seismic statistics indicates that in background seismicity, the duration of the failure cycle does not depend (or depends very weakly) on the size of the fracture area.

From the standpoint of physics of fracture, the inverse to the duration of failure cycle-the mean rate of fracture events damaging the same domain of size $l$ of the medium-characterizes the average probability of failure of the domain of size $l$ of the medium. This probability can be calculated within some or other fracture theory. The particular formulas will differ depending on the selected approach (the concept of criticality for a system of cracks, kinetic concept of fracture, etc.). However, in any case, the probability of fracture is determined by the ratio between the strength properties and the acting stresses. The statistical result $q \approx 0$ indicates that in regard to the lithosphere, the long-term values of the probability of failure do not depend on the size of the fracturing region. This means that the long-term stress field is matched with the strength field in such a way that the different size elements of the medium have approximately equal probability to fracture.

\section{Deviations of $q$-Value from the Mean}

The analysis of variance conducted in this work for spatial variations in $q$-value on the regional level has shown that standard deviation of these variations is larger than the error of statistical estimation of this parameter. This means that relation (12) which is valid on average, is locally violated in some regions of the lithosphere. 
The mean absolute $q$-value based on the data of the worldwide earthquake catalogue is 0.45 (Smirnov, 2003). The mean absolute $q$ estimated from regional catalogues ranges within $0.4-0.7$ which is consistent with the range of the values of serial variances (Tables $1-3$ ). The greater variability of $q$-value on the regional level compared to the global level is likely to be due to the smaller cell of spatial averaging on the regional level. As the characteristic deviation of $q$-value from the zero mean we use $q=0.5$.

In (Smirnov, 2003), based on the Zhurkov's formula (1968)

$$
\tau=\theta_{0} e^{\frac{U_{0}-\gamma \sigma}{k T}},
$$

where $\theta_{0}$ is the period of thermal oscillations, $k$ is Boltzmann constant, $T$ is temperature, $U_{0}$ is the bond dissociation energy, $\gamma$ is the Zhurkov's structure-sensitive parameter, and $\sigma$ are macroscopic stresses, the author estimated the degree of nonuniformity of stress distribution across lithospheric inhomogeneities that ensures nonzero $q$-values under the assumption that the strength is uniformly distributed across the hierarchical levels of the system of defects of the geophysical medium. For the difference of the stresses $\sigma_{2}$ and $\sigma_{1}$ acting on the elements with sizes $l_{2}$ and $l_{1}$, respectively, the following expression was obtained:

$$
\Delta \sigma=\sigma_{2}-\sigma_{1}=\frac{k T}{\gamma} q \ln \frac{l_{2}}{l_{1}} .
$$

If we assume that parameter $\gamma$ ranges within $1000-$ $4000 \mathrm{~J} /$ mole $\mathrm{MPa}$ as established by the laboratory experiments (Stavrogin and Tarasov, 2001) and postulate that $\gamma$ does not depend on the scale of fracture, at $q=0.5$ we find from (14) for the neighboring hierarchical levels according to Sadovskii ${ }^{7}$ (at $l_{2} / l_{1}=3$ ): $\Delta \sigma=0.3-1 / 2 \mathrm{MPa}($ at $T=300 \mathrm{~K}$ ). These values are commensurate with the characteristic stress drop by the earthquakes (Earthquakes..., 2000) which supports the hypothesis of the probable stress variations on this order of magnitude across the hierarchical levels.

In (Smirnov, 2003) it was noted that constancy of the structure-sensitive parameter $\gamma$ which is assumed in the estimation according to (14) is conditional. The dependence of this parameter on the scale of fracture has been rather poorly explored in the experiments (Regel et al., 1974; Stavrogin and Tarasov, 2001). Smirnov, 2003) it was pointed out that the existence of this dependence can be expected if $\gamma$ is understood as a coefficient of overload (or overstress) characterizing the microscopic to macroscopic stress ratio (Regel, 1974). This ratio can depend, inter alia, on the pres-

\footnotetext{
${ }^{7}$ The applicability of this relation for seismicity was demonstrated, inter alia, in (Smirnov and Ispolinova, 1995) where a logarithmically uniform polymodality of deviations of seismic moment distribution for the worldwide earthquakes from the Gutenberg-Richter law was revealed.
}

ence of cracks, and in the case of the elongated Griffith-type crack it is proportional to the square root of its length. For a system of cracks with overlapping stress fields (i.e., in the case of fairly high crack density), one should expect even stronger dependence. Since with the increase in the size of the domain in a medium, the probability of the presence of larger cracks therein increases and so does the coefficient of overload, parameter $\gamma$ can also be expected to grow with the increase of the domain size. This is consistent with the decrease in strength with the increase of specimen size known from the experiments (Scholz, 2019).

In this case, the independence of the probability of fracture for the elements of the lithosphere on their size which is revealed from the data of seismic catalogues indicates that in the consistent state when relation (12) holds, the stresses (within the kinetic concept of fracture) or the rate of the stress growth (within the crack theory) are nonuniformly distributed across sizes of the defects of the medium: the stresses or their rate of growth on the smaller defects is higher than on the larger defects. In this case, the estimates by formula (14) should be considered as deviations from this nonuniform basic distribution.

We could not find the direct measurement data on stress distribution on different scale defects of the medium (in the natural or laboratory conditions). However, the growth of stresses stress with the transition to the microscopic scales is supported by the experimental studies of G.A. Sobolev et al. on fracture of the rock samples with monitoring the parameters of crystalline lattice by neutron tomography (Sobolev et al., 2004; Nikitin et al., 2004).

\section{Failure Cycle Duration $\tau_{0}$}

The analysis of the obtained set of estimates for $\tau_{0}$ shows that $70 \%$ of the $\tau_{0}$ values are within 300 to 30000 years. This range agrees with the existing direct estimates on the duration of seismic cycle by the seismological, paleoseismological, and geological methods: in the different regions and according to the different authors, the durations are hundreds, thousands, and tens of thousands years (Scholz, 2002; Grant, 2015).

In (Smirnov, 2003) we hypothesized that spatial variations in failure cycle duration can probably be due to the corresponding variations in the properties and the stress state of the lithosphere. If we assume that mechanical properties of the lithosphere do not vary greatly (at most severalfold) along the active seismic belts, then the variations in $\tau_{0}$ by several orders of magnitude should be explained by the differences in the strain rates by several orders of magnitude which is not inconsistent with the probable range of regional differences in the tectonic strain rates (Kukal, 1987).

Let us hypothesize that the duration of the failure cycle is determined, on a coarse approximation, by the strength of the medium and by the strain rate 


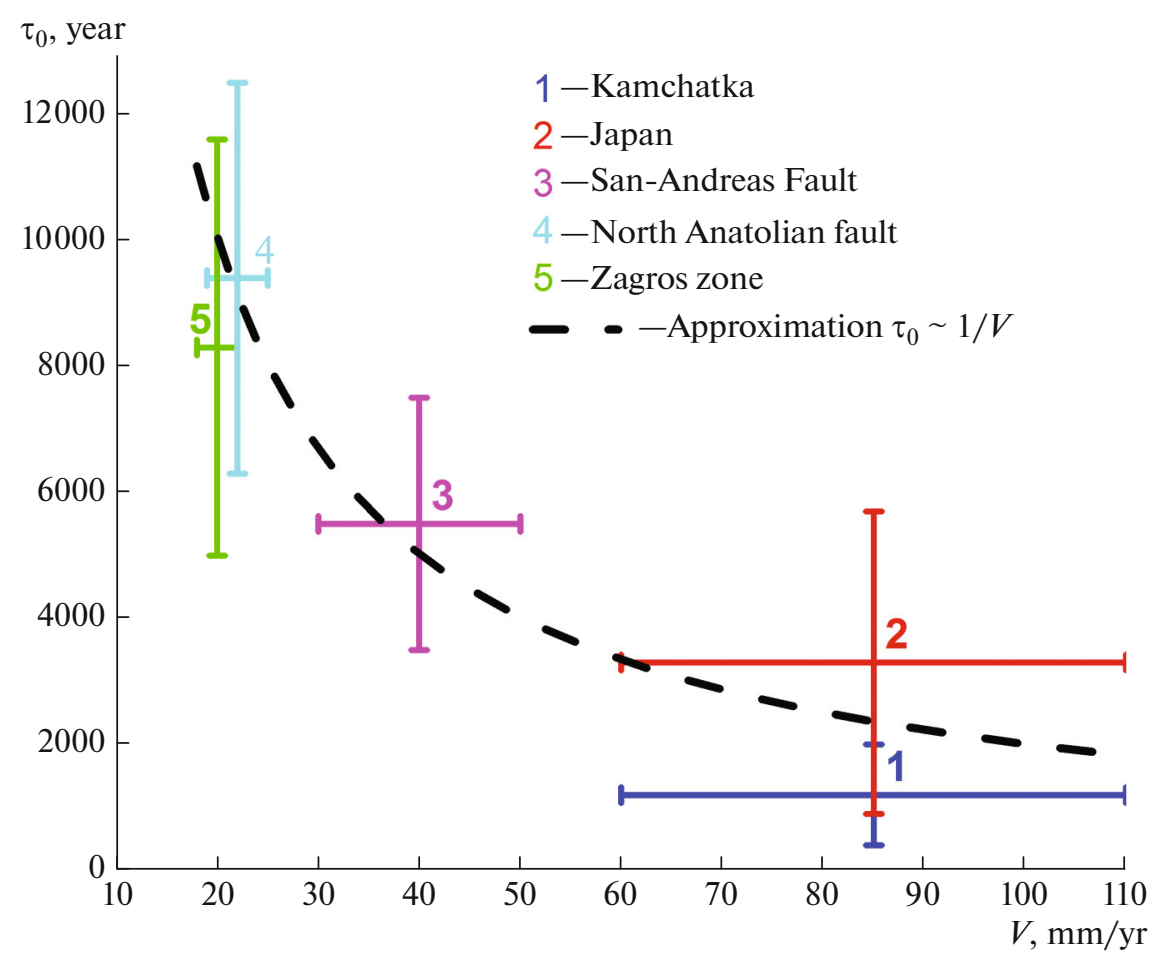

Fig. 9. Duration of failure cycle of lithosphere as function of velocity of relative plate motions.

$\tau_{0} \approx \varepsilon^{*} / \dot{\varepsilon}$, where $\varepsilon^{*}$ is deformation strength. Assuming that the ultimate stress on the fault is close by the order of magnitude to the stress drop, we obtain the following estimate for $\varepsilon^{*}: \varepsilon^{*} \approx \Delta \sigma / \mu \approx 10^{-4}$. The characteristic $\dot{\varepsilon}$ values in the active tectonic regions are $10^{-7} 1 / \mathrm{yr}\left(3 \times 10^{-15} 1 / \mathrm{s}\right)$. Therefore, the estimate for the characteristic $\tau_{0}$ values is $10^{3}$ years which is consistent on the order of magnitude with our estimates for the duration of the failure cycle.

The modern data provide the possibility to quantitatively compare, on a regional scale, the duration of the failure cycle with the rate of tectonic deformations. We opted to characterize the latter in terms of the average velocity of relative plate motions in the regions with the clearly pronounced tectonic structures of the transform faults: in the subduction zone and in the shear zones.

The data on the velocities of recent motions for the regions selected for our study were taken from the published sources. For all regions this is the GPS data, in some cases combined with the results of ground-based geodetic measurements. The regions selected for our analysis are well explored, the results are extensively covered in regional publications and summarized in the monographs and textbooks (Turcotte and Schubert, 2002; Papazachos and Papazachou, 1977; Stein and Wysession, 2003; Fowler, 2005).
The comparison of the regional estimates for the duration of failure cycle with the velocities $V$ of relative plate motions is illustrated in Fig. 9. The whiskers error bars in the graphs show serial deviations (square root of serial variance) which are double to triple the statistical estimation errors and, thus, reflect the real scatter (range) of the values within each region. In the figure it can be seen that despite the significant amplitude of this scatter, the empirical data are reasonably well fitted by the dependence $\tau_{0} \propto 1 / V$.

Thus, in the first approximation it can be deemed that region mean duration of the failure cycle of the lithosphere is mainly determined by the velocity of tectonic deformations. However, as noted above, even within relatively small regions considered in this study the $\tau_{0}$ values vary within two orders of magnitude. This testifies to the substantial inhomogeneity of the intraregional distribution of the strain rates and strength and empirically supports the modern notions of the hierarchical structures of the geological medium and nonuniform mosaic pattern of the stress field.

\section{CONCLUSIONS}

The generalized Gutenberg-Richter law has been published in explicit form independently by two groups of authors (Kossobokov and Mazhkenov, 1988; Chelidze, 1990). Keilis-Borok et al. (1988) were first to obtain estimates for the waiting times between the 
successive earthquakes reduced to their source area, averaged over several regions.

In 2002, Bak et al. (2002), in fact, rediscovered the generalized Gutenberg-Richter law for earthquakes and named it the unified scaling law for earthquakes. The further development of Bak's ideas is associated with rather rare works mainly by mathematicians and physicists (Corral, 2003; 2004; 2005; Carbone et al., 2005; Davidsen and Goltz, 2004; Lindman et al., 2005; Livina et al., 2005; Shcherbakov et al., 2006). In fact, these works concern the existence of the statistical selfsimilarity of seismicity in space-time-energy; the relationship is presented between the self-similarity exponents in space (fractal dimension), in time, and in the energy domain (the Gutenberg-Richter parameter) which provides this matched self-similarity. This is equivalent to the statement about the equality to zero of the parameter $q$ introduced by us (Smirnov, 2003).

The estimates of the parameters of failure cycle on the regional level indicate that in the background regime (with averaging over decades), the duration of the failure cycle of the lithosphere does not depend on the size of the fracture region: the $q$-value is close to zero. This means that in the background regime, the fracture process on different spatial scales develops with approximately the same intensity; i.e., the probability of fracture for the elements of the medium is uniformly distributed across their sizes. This situation is observed when the stress field is matched in a certain way with the strength field. In this case, the distribution of the earthquakes by energies (the GutenbergRichter frequency-magnitude relationship) is exclusively determined by the geometry of the structure of the system of lithospheric inhomogeneities (assuming that this geometry is fractal). Parameter $q$ characterizes the difference in the scalings (exponents) of statistical distributions of the sizes of seismic sources and the distances between earthquakes. The closeness of the $q$-value to zero means that these scaling in the background regime are close to each other and the mean distance between the earthquakes is proportional to the source size (Smirnov and Ponomarev, 2004).

Spatial variations in the $q$-value can probably be associated with the nonuniform distribution of stresses or the rates of their growth across differently sized inhomogeneities of the lithosphere. This hypothesis agrees with the significant deviations of $q$ from zero in the time variations during transitional regimes of the seismic process when the stress field and, perhaps, also the strength of the medium locally substantially vary (Smirnov and Ponomarev, 2004; Bayrak et al., 2017; Smirnov et al., 2018). .

The region-mean duration of the failure cycle of the lithosphere is mainly determined by the velocity of tectonic deformations. Moreover, the significant range of variations in the duration of the failure cycle within relatively small regions testifies to the considerably nonuniform distribution of the strain rates and strength within the region, consistent with the modern notions of the hierarchical nature of a geological medium and mosaicity of the stress field.

\section{FUNDING}

The work was supported by the Megagrant from the Ministry of Science and Education of the Russian Federation under project no. 14.W03.31.0033 "Geophysical studies, monitoring, and forecasting the development of catastrophic geodynamical processes in the Far East of the Russian Federation.”

\section{REFERENCES}

Abubakirov, I.R., Gusev, A.A., Guseva, E.M., Pavlov, V.M., and Skorkina, A.A., Mass determination of moment magnitudes $M_{\mathrm{w}}$ and establishing the relationship between $M_{\mathrm{w}}$ and $M_{L}$ for moderate and small Kamchatka earthquakes, Izv., Phys. Solid Earth, 2018, vol. 54, no. 1, pp. 33-47.

Aki, K., A probabilistic synthesis of precursory phenomena, in Earthquake Prediction: An International Review, vol. 4, Washington: AGU, 1981, pp. 556-574.

Bak, P., Christensen, K., Danon, L., and Scanlon, T., Unified scaling law for earthquakes, Phys. Rev. Lett., 2002, vol. 88 , no. 17.

https://doi.org/10.1103/PhysRevLett.88.178501

Bayrak, E., Yilmaz, S., and Bayrak, Y., Temporal and spatial variations of Gutenberg-Richter parameter and fractal dimension in Western Anatolia, Turkey, J. Asian Earth Sci., 2017, vol. 138, no. 1, pp. 1-11.

Carbone, V., Sorriso-Valvo, L., Harabaglia, P., and Guerra, I., Unified scaling law for waiting times between seismic events, Europhys. Lett., 2005, vol. 71. no. 6, pp. 1036-1042. Chelidze, T.L., Generalized fractal law of seismicity, Dokl. Akad. Nauk SSSR, 1990, vol. 314, no. 5, pp. 1104-1105

Corral, A., Local distributions and rate fluctuations in a unified scaling law for earthquakes, Phys. Rev. E, 2003, vol. 68, 035102(R).

Corral, A., Long-term clustering, scaling, and universality in the temporal occurrence of earthquakes, Phys. Rev. Lett., 2004, vol. 92, no. 10, 108501-1.

Corral, A., Renormalization-group transformations and correlations of seismicity, Phys. Rev. Lett., 2005, vol. 95. https://doi.org/10.1103/PhysRevLett.95.028501

Davidsen, J. and Goltz, Ch., Are seismic waiting time distributions universal?, Geophys. Res. Lett., 2004, vol. 31, L21612.

https://doi.org/10.1029/2004GL020892

Earthquakes: Radiated Energy and the Physics of Faulting, Abercombie, R., McGarr, A., Di Toro, G., and Kanamori, H., Eds., vol. 170 of Geophysical Monograph Series, Washington: AGU, 2000.

Fedotov, S.A., On seismic cycle, possibility of quantitative seismic zoning, and long-term seismic forecasting, in Seismicheskoe raionirovanie SSSR (Seismic Zoning of the USSR), Moscow: Nauka, 1968, pp. 121-150.

Fowler, C.M.R., The Solid Earth: An Introduction to Global Geophysics, Cambridge: Cambridge Univ. Press, 2005.

Gordeev, E.I., Fedotov, S.A., and Chebrov, V.N., Main results of seismological studies in Kamchatka based on the 
detailed observations from 1961 to 2011, in Seismologicheskie i geofizicheskie issledovaniya na Kamchatke. K 50-letiyu detal'nykh seismologicheskikh nablyudenii (Seismological and Geophysical Studies in Kamchatka: To the 50th Anniversary of the Detailed Seismological Observations), Petropavlovsk-Kamchatskii, 2012, pp. 9-35.

Gordeev, E.I., Fedotov, S.A., and Chebrov, V.N., Detailed seismological investigations in Kamchatka during the 1961-2011 period: main results, J. Volcanol. Seismol., 2013, vol. 7 , no. 1 , pp. $1-15$.

Grant, L.L., Paleoseismology, Chapter 4.21 of Volume 4: Earthquake Seismology, in Treatise on Geophysics (Second Edition), Schubert, G., Editor-in Chief, 2015, pp. 559-579.

Grigoryan, S.S., On the mechanism of emergence of an earthquake and on the meaning of empirical relationships of seismology, Dokl. Akad. Nauk SSSR, 1988, vol. 299, no. 5.

Gusev, A.A. and Melnikova, V.N., Relationships between magnitude scales for global and Kamchatkan earthquakes, Volcanol. Seismol., 1992, vol. 12, p. 723-733.

Keilis-Borok, V.I., Kosobokov, V.G., and Mazhkenov, S.A., On similarity in the spatial distribution of seismicity, in $V y$ chislitel'naya Seismologiya (Computational Seismology), Moscow, 1989, vol. 22, pp. 28-40.

King, G., The accommodation of large strain in the upper lithosphere of the Earth and other solids by self-similar fault system: the geometrical origin of $b$-value, Pure Appl. Geophys., 1983, vol. 121, pp. 761-815.

Kossobokov, V.G. and Mazhkenov, S.A., Spatial Characteristics of Similarity for Earthquake Sequences: Fractality of Seismicity, Lecture Notes of the Workshop on Global Geophysical Informatics Application to Research in Earthquake Prediction and Reduction of Seismic Risk, November 15December 16, 1988, Trieste: ICTP, 1988.

Kosobokov, V.G. and Mazhkenov, S.A., On similarity in the spatial distribution of seismicity, in Computational Seismology and Geodynamics, vol. 1, Selected Papers from Volumes 22 and 23 of Vychislitel'naya Seismologiya, Washington: AGU, 1994, pp. 6-15.

Kossobokov, V.G. and Nekrasova, A.K., General similarity law for the esrthquakes: global map of parameters, in $V y$ chislitel'naya seismologiya (Computational Seismology), Moscow: Nauka, 2004, vol. 35, pp. 160-176.

Kossobokov, V.G. and Soloviev, A.A., Pattern recognition in seismic hazard assessment, Chebyshevskii Sb., 2018, vol. 19 , no. 4 , pp. 55-90.

Kukal, Z., Rychlost Geologických Procesů, Praha: Academia, 1983.

Lindman, M., Jonsdottir, K., Roberts, R., Lund, B., and Bodvarsson, R., Earthquakes descaled: on waiting time distributions and scaling laws, Phys. Rev. Lett., 2005, vol. 94, no. $10,108501$.

Livina, V.N., Havlin, S., and Bunde, A., Memory in the occurrence of earthquakes, Phys Rev Lett., 2005, vol. 95, no. 20, 208501 .

Molchan, G.M. and Dmitrieva, O.E., Identification of aftershocks: a review and new approaches, in Vychislitel'naya Seismologiya (Computational Seismology), Moscow: Nauka, 1991, vol. 24, pp. 19-50.

Molchan, G.M. and Dmitrieva, O.E., Targeted approach to the aftershock identification problem, in Seismichnost' $i$ seismicheskoe raionirovanie Severnoi Evrazii (Seismicity and
Seismic Zoning of North Eurasia), vol. 1, Moscow: IFZ RAN, 1993, pp. 62-69.

Molchan, G.M., Kronrod, T.L., Dmitrieva, O.E., and Nekrasova, A.K., Hazard-oriented multiscale seismicity: Italy, in Selected Papers from Volumes 28 and 29 of "Vychislitel'naya Seismologiya," vol. 4, Computational Seismology and Geodynamics Book Series, vol. 4, Washington: AGU, 1996, pp. 138-156.

Nekrasova, A.K., General law of similarity for earthquakes: Application to seismically active regions of the world, Doctoral (Phys.-Math.) Dissertation, Moscow: Inst. of Earthquake Prediction Theory and Math. Geophys., Russ. Acad. Sci., 2008.

Nekrasova, A.K. and Kosobokov, V.G., Temporal variations in the parameters of the unified scaling law for earthquakes in the eastern part of Honshu Island (Japan), Dokl. Earth Sci., 2005, vol. 405a, no. 9, pp. 1352-1355.

Nekrasova, A.K. and Kosobokov, V.G., General law of similarity for earthquakes: evidence from the Baikal region, Dokl. Earth Sci., 2007, vol. 407a, no. 3, pp. 484-486.

Nekrasova, A., Kossobokov, V., Peresan, A., Aoudia, A., and Panza, G.F., A multiscale application of the unified scaling law for earthquakes in the Central Mediterranean Area and Alpine Region, Pure Appl. Geophys., 2011, vol. 168, pp. 297-327.

Nekrasova, A.K., Kosobokov, V.G., and Parvez, I.A., Seismic hazard and seismic risk assessment based on the unified scaling law for earthquakes: Himalayas and adjacent regions, Izv., Phys. Solid Earth, 2015, vol. 51, no. 2, pp. 268-277.

Nikitin, A.N., Ivankina, T.I., Sobolev, G.A., Scheffzük, Ch., Frischbutter, A., and Walther, K., Neutron diffraction study of intracrystalline strains and stresses in a marble sample at high temperatures and external mechanical loads, Izv., Phys. Solid Earth, 2004, vol. 40, no. 1, pp. 83-87.

Papazachos, B. and Papazachou, C., The Earthquakes of Greece, Thessaloniki: Ziti, 1997.

Pisarenko, V.F., On the magnitude-frequency distribution of earthquakes, in Diskretnye svoistva geofizicheskoi sredy (Discrete Properties of a Geophysical Medium), Moscow: Nauka, 1989, pp. 47-60.

Regel', V.R., Slutsker, A.I., and Tomashevskii, E.E., Kineticheskaya priroda prochnosti tverdykh tel (Kinetic Nature of the Strength of Solids), Moscow: Nauka, 1974.

Sadovskii, M.A. and Pisarenko, V.F., Seismicheskii protsess $v$ blokovoi srede (Seismic Process in a Block Medium), Moscow: Nauka, 1991.

Sadovskii, M.A., Pisarenko, V.F., and Shteinberg, V.V., On the dependence of earthquake energy on the volume of seismic source, Dokl. Akad. Nauk SSSR, 1983, vol. 271, no. 3, pp. 598-602.

Scholz, C.H., The Mechanics of Earthquakes and Faulting, Cambridge: Cambridge Univ. Press, 2019.

Shcherbakov, R., Turcotte, D.L., and Rundle, J.B., Scaling properties of the Parkfield aftershock sequence, Bull. Seismol. Soc. Am., 2006, vol. 94. pp. 376-384.

https://doi.org/10.1785/0120050815

Smirnov, V.B. and Ispolinova, S.I., On the discrete energy structure of seismicity, Dokl. Akad. Nauk, 1995, vol. 342, no. 6, pp. 809-811.

Smirnov, V.B., Spatial and temporal variations of fractal properties of seismicity, Volcanol. Seismol., 1998, vol. 19, pp. 805-818. 
Smirnov, V.B., Earthquake recurrence and seismicity parameters, Volcanol. Seismol., 1995, vol. 17, pp. 307-319.

Smirnov, V.B., Earthquake catalogs: evaluation of data completeness, Volcanol. Seismol., 1998, vol. 19, pp. 497510.

Smirnov, V.B., Estimating the duration of the lithospheric failure cycle from earthquake catalogs, Izv., Phys. Solid Earth, 2003, vol. 39, no. 10, pp. 794-811.

Smirnov, V.B., Prognostic anomalies of seismic regime. I. Technique for preparation of original data, Geofiz. Issled., 2009, vol. 10, no. 2, pp. 7-22.

Smirnov, V.B. and Ponomarev, A.V., Seismic regime relaxation properties from in situ and laboratory data, Izv., Phys. Solid Earth, 2004, vol. 40, no. 10, pp. 807-816.

Smirnov, V.B. and Zaviyalov, A.D., Incorporating the fractal distribution of faults as a measure of failure concentration, Volcanol. Seismol., 1997, vol. 18, pp. 447-452.

Smirnov, V.B., Ponomarev, A.V., Kartseva, T.I., Mikhailov, V.O., Chadha, R.K., and Aidarov, F., Dynamics of induced seismicity during the filling of the Nurek reservoir, Izv., Phys. Solid Earth, 2018, vol. 54, no. 4, pp. 641-652.

Sobolev, G.A., Ponomarev, A.V., Nikitin, A.N., Balagurov, A.M., and Vasin, R.N., Dynamics of the polymorphic $\alpha-\beta$-transition in quartzite from data of neutron diffractometry and acoustic emission, Izv., Phys. Solid Earth, 2004, vol. 40, no. 10, pp. 788-797.

Stakhovsky, I.R., A model of scaling correspondence between fault and seismic fields, Izv., Phys. Solid Earth, 2001, vol. 37, no. 7, pp. 547-555.

Stakhovsky, I.R., Model of scaling correspondence between fault and seismic fields of the Earth's crust: some generalizations, Izv., Phys. Solid Earth, 2004a, vol. 40, no. 9 , pp. 745-751.

Stakhovsky, I.R., Interrelation between spatial and energy scalings of the seismic process, Izv., Phys. Solid Earth, 2004b, vol. 40, no. 10, pp. 840-848.

Stakhovskii, I.R., Scale invariance of shallow seismicity and the prognostic signs of earthquakes Phys.-Usp., 2017, vol. 60 , no. 5, pp. 472-489.

Stavrogin, A.N. and Tarasov, B.G., Eksperimental'naya fizika i mekhanika gornykh porod (Experimental Physics and Mechanics of Rocks), St. Petersburg: Nauka, 2001.

Stein, S. and Wysession, M., An Introduction to Seismology, Earthquakes, and Earth Structure, Malden: Blackwell, 2003.

Turcotte, D.L., Fractals and Chaos in Geology and Geophysics, Cambridge: Cambridge Univ., 1992.

Turcotte, D. and Schubert, G., Geodynamics, Cambridge: Cambridge Univ., 2002.

Ulomov, V.I., A lattice model of source seismicity and seismic hazard forecasting, Uzb. Geolog. Zh., 1987, no. 6, pp. 20-25.

Ulomov, V.I., Global ordering of seismogeodynamic structures and some aspects of seismic zoning and long-term earthquake forecasting, in Seismichnost' $i$ seismicheskoe raionirovanie Severnoi Evrazii (Seismicity and Seismic Zoning of North Eurasia), vol. 1, Moscow: IFZ RAN, 1993, pp. 24-44.

Zhurkov, S.N., Kinetic concept of the strength of solids, Vestn. Akad. Nauk SSSR, 1968, no. 3, pp. 46-52.

Translated by M. Nazarenko 